\title{
Electrostatic sensor modeling for torque measurements
}

\author{
Michał Mika ${ }^{1}$, Mirjam Dannert ${ }^{1}$, Felix Mett ${ }^{3}$, Harry Weber $^{2}$, Wolfgang Mathis ${ }^{2}$, and Udo Nackenhorst ${ }^{1}$ \\ ${ }^{1}$ Institut für Numerische Mechanik, Leibniz Universität Hannover, Appelstraße 9A, 30167 Hannover, Germany \\ ${ }^{2}$ Institut für Theoretische Elektrotechnik, Leibniz Universität Hannover, Appelstraße 9A, 30167 Hannover, Germany \\ ${ }^{3}$ Leibniz Universität Hannover, Welfengarten 1, 30167 Hannover, Germany
}

Correspondence to: Michał Mika (mika@ stud.uni-hannover.de)

Received: 28 December 2016 - Accepted: 9 April 2017 - Published: 21 September 2017

\begin{abstract}
Torque load measurements play an important part in various engineering applications, as for automotive industry, in which the drive torque of a motor has to be determined. A widely used measuring method are strain gauges. A thin flexible foil, which supports a metallic pattern, is glued to the surface of the object the torque is being applied to. In case of a deformation due to the torque load, the change in the electrical resistance is measured. With the combination of constitutive equations the applied torque load is determined by the change of electrical resistance. The creep of the glue and the foil material, together with the temperature and humidity dependence, may become an obstacle for some applications (Kapralov and Fesenko, 1984). Thus, there have been optical and magnetical, as well as capacitive sensors introduced (Turner, 1988; Wolffenbuttel and Foerster, 1990). This paper discusses the general idea behind an electrostatic capacitive sensor based on a simple draft of an exemplary measurement setup. For better understanding an own electrostatical, geometrical and mechanical model of this setup has been developed.
\end{abstract}

\section{Introduction}

The state of the art method for the measuring of mechanical torque loads are strain gauges. These are thin flexible foils glued to the surfaces of an object, that change the electrical resistance due to deformation. Their application for engineering purposes is cheap, simple and well established. However, the measurement accuracy depends on the humidity and the surrounding temperature. Also, the creep of the foil and the glue contributes to the measurement error (Kapralov and Fesenko, 1984). To address these problems an electrostatic capacitive sensor is being proposed. Similar concepts have been already introduced in the works of Turner (1988) and Wolffenbuttel and Foerster (1990).

\section{Measuring setup and operation principle}

As shown in Fig. 1, the sensor consists of a cantilever beam with a circular cross-section over an ideal conducting plate. A thin, elastic electret foil with a constant surface charge density is attached to the bottom side of the beam. In comparison to the electret the plate is assumed to have much bigger dimensions. The beam itself and its mounting are made out of polyethylene. Due to the permittivity of polyethylene, $\varepsilon_{\text {pet }} \approx 1$, these can be ignored in the electrical modeling. The charge leads to an electrostatic field $\boldsymbol{E}$ and the related electric displacement field $\boldsymbol{D}$. This induces an electric charge on the plate, which can be measured. As a result of the torque load $M_{\mathrm{t}}$, the beam deforms, which leads to a changed position of the electret relative to the height of the plate. Thus, the induced charge on the plate changes. This change can be associated with the equivalent torque load. In the following the relation between the torque load and the measured charge is being discussed, by modeling the system in Matlab and performing a numerical analysis for different torque loads. First, the problem is being approached by analyzing the mechanical behavior of the beam.

\section{Model geometry and torsional mechanics}

In order to model the behavior of an electrostatic field, it is important to understand the model geometry and to define the mechanical assumptions for a beam with a torque load $M_{\mathrm{t}}$ at the end of the beam. The beam deformation dictates the deformation of the electret, therefore it describes the displace- 


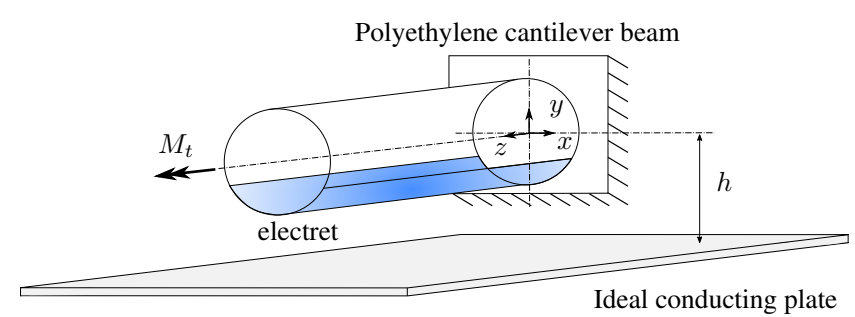

Figure 1. Measuring setup consisting of the beam, electret and the ideal conducting plate.

ment of the electric charge in the space, when a torque load is applied. For the St. Vénant torsion theory (Szabó, 2001) the fundamental assumption is a constant twist rate $\frac{\vartheta}{l}$ over the length of the beam. Furthermore, there is no distortion of the cross-sections in $x$ and $y$ direction. The cross-sections rotate as rigid bodies around the beam axis. Also, the warping of the cross-section is allowed, though it remains equal over the length of the beam. Furthermore, the electret is being ignored in this modeling, because it is thin and elastic enough, not to contribute to the overall stiffness of the beam. From the mathematical perspective the cross-sections have to be a connected space with a boundary $R$. The shear stress component $\sigma_{z t}$ is tangent to the boundary $R$.

Assuming the shear angle $\gamma \ll 1$ as shown in Fig. 2a, it is possible to describe the twist angle at $z$ as

$\vartheta_{z}=\frac{\vartheta}{l} z$

As shown in Fig. $2 \mathrm{~b}$, the motion of a point $P$ depends on the twist angle $\vartheta_{z}$ as follows

$x+v=r \cos \left(\beta+\vartheta_{z}\right), \quad y+w=r \sin \left(\beta+\vartheta_{z}\right)$.

Using the trigonometric identities and assuming small deformations, the linear approximation of motion of $P$ in relation to the twist angle $\vartheta$ at the end of the beam is described by

$x+v=r \cos \beta-r \vartheta_{z} \sin \beta=x-\frac{\vartheta}{l} y z$

$y+w=r \sin \beta+r \vartheta_{z} \cos \beta=y+\frac{\vartheta}{l} x z$.

The displacements are

$v=-\frac{\vartheta}{l} y z, \quad w=\frac{\vartheta}{l} x z$.

Based on the St. Vénant assumption of constant cross-section warping, the deformation along the beam axis can be formulated as

$u(x, y, z)=\frac{\vartheta}{l} \varphi(x, y) \quad \forall z \in[0 ; l]$.

Leaving the warping function $\varphi(x, y)$ unknown for the time being, it is easy to see, that the warping of the cross-section for small twist rates $\frac{\vartheta}{l}$ also remains small.

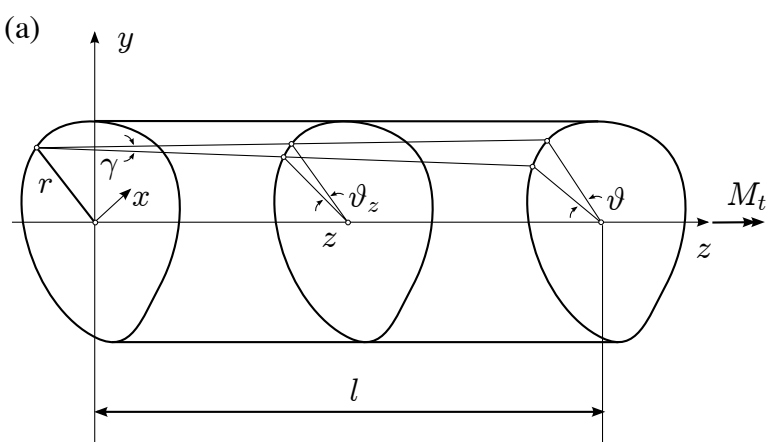

(b)

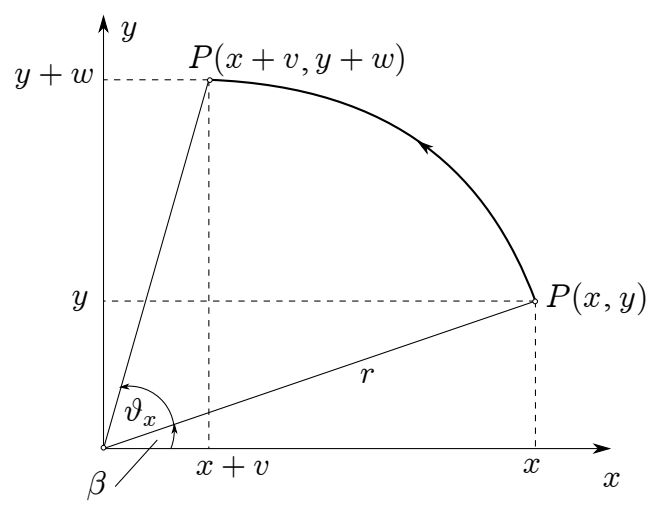

Figure 2. Torsional deformation of the beam. Figures adapted from Szabó (2001). (a) Twist of the beam along the $z$-axis. (b) Movement of a point $P(x, y)$ in the cross-section plane as a result of torsional beam deformation.

\section{Explicit formulation of the point motion}

Foregoing the derivation of the St. Vénant torsion theory, the torsion rate at the end is defined as

$D=\frac{\vartheta}{l}=\frac{M_{t}}{G J_{\mathrm{t}}}$,

where $G$ is the shear modulus and the $J_{\mathrm{t}}$ describes the torsional second moment of area (Szabó, 2001), which for circular beam cross-sections equals

$J_{\mathrm{t}}=\int_{\mathcal{A}} r^{2} \mathrm{~d} A=\frac{1}{2} \pi r^{4}$.

Using the described geometry and the St. Vénant torsion theory, it is possible to formulate a simple geometrical model of the small deformations as shown in Fig. 3

$\Gamma=\left[\begin{array}{c}\Gamma_{x} \\ \Gamma_{y} \\ \Gamma_{z}\end{array}\right]=\left[\begin{array}{c}r \cos \left(\varphi+\frac{\vartheta}{l} z\right) \\ r \sin \left(\varphi+\frac{\vartheta}{l} z\right) \\ z\end{array}\right]$

where $\varphi \in\left[\frac{11}{8} \pi ; \frac{13}{8} \pi\right]$ and $z \in[0 ; l]$. 


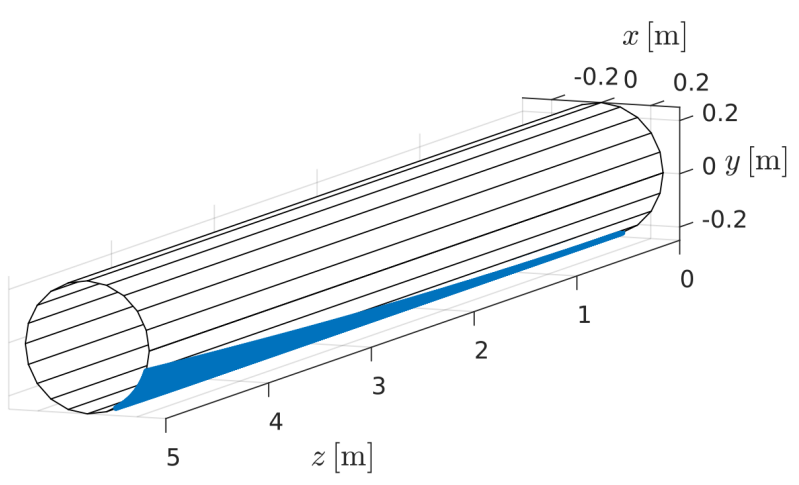

Figure 3. Simple geometrical model of the charge holding particles. For a linear approximation the twist rate follows from Eqs. (1), (7) and (8).

\section{Mathematical modeling of the electrostatic field}

The presence of a static charge holding particle results in a physical electrostatic field (Küpfmüller et al., 2013). The relation of the charge density $\varrho(r)$ to the electric displacement field $\boldsymbol{D}$ is described by

$\operatorname{div} \boldsymbol{D}=\varrho(\boldsymbol{r})$.

Furthermore, based on the experiments by Coulomb and Cavendish, the electrostatic field $\boldsymbol{E}$ is defined as the force on the charge holding particle normalized by the holden charge $q$,

$\boldsymbol{E}=\frac{\boldsymbol{F}}{q}$.

Observations of $\boldsymbol{D}$ lead in first order to a linear isotropic constitutive equation

$\boldsymbol{D}=\varepsilon \boldsymbol{E}$.

with the constant permittivity $\varepsilon$.

Based on the experiments, it is known that the forces on the particle are central forces. Thus, the curl of the described fields has to be equally zero,

$\operatorname{rot} \boldsymbol{F}=q \operatorname{rot} \boldsymbol{E}=\varepsilon q \operatorname{rot} \boldsymbol{D}=0$.

Therefore, the physical electrostatic field can be mathematically described by

$\operatorname{div} \boldsymbol{D}=\varrho(\boldsymbol{r}), \quad \boldsymbol{D}=\varepsilon \boldsymbol{E}, \quad \operatorname{rot} \boldsymbol{E}=0$.

Following these equations, a scalar electrostatic field potential $\varphi: \mathbb{R}^{3} \rightarrow \mathbb{R}$ is being defined as

$\boldsymbol{E}=:-\operatorname{grad} \varphi$.

This is possible without loss of generality, because of the curl-free characteristic of the described fields. In general, the condition $\operatorname{rot}(\operatorname{grad}(\cdot))=0$ is satisfied, therefore the potential fields are curl-free.

Furthermore, the divergence of the electric displacement field is used to derive an elliptic partial differential equation

$\operatorname{div} \boldsymbol{D}=\operatorname{div}(-\varepsilon \cdot \operatorname{grad} \varphi)=-\varepsilon \cdot \operatorname{div}(\operatorname{grad} \varphi)=\varrho(\boldsymbol{r})$.

Thus, the electrostatic field can be described by a Poisson equation of the field potential $\varphi$

$\Delta \varphi=\frac{\partial^{2} \varphi}{\partial x^{2}}+\frac{\partial^{2} \varphi}{\partial y^{2}}+\frac{\partial^{2} \varphi}{\partial z^{2}}=-\frac{\varrho(\boldsymbol{r})}{\varepsilon}$.

\section{Kirchhoff's Integral Theorem}

In the case of homogeneous potential field problems, in which the only boundary condition is postulated by the decrease of the field over the distance and eventually vanishing at an infinite distance, there are known analytic solutions to the elliptic partial differential, e.g. Kirchhoff's integral theorem based on Greens identities (Küpfmüller et al., 2013),

$\varphi(\boldsymbol{r})=\frac{1}{4 \pi \varepsilon} \iint_{\mathcal{V}} \frac{\varrho(\widetilde{\boldsymbol{r}})}{\|\boldsymbol{r}-\widetilde{\boldsymbol{r}}\|} \mathrm{d} V$.

Considering the measuring setup in Fig. 4, the Kirchhoff's integral can not be applied. The plate beneath the electret postulates an inhomogeneous boundary condition.

However, there is a possibility to substitute the current setup with an alternative setup just for the computation purpose. The first point to consider, is the fact that the potential $\varphi$ at the plate has a constant value. This is a consequence of the assumption of the electrostatic field theory. The static characteristic of the electrons shall not be violated, therefore no potential differences in the ideal conducting plate are allowed. Furthermore, because of the fact that the charge influence decreases with the distance to the charge itself, the potential at the plate in a certain sufficient distance from the charge equals zero. The plate is assumed as infinitely large, therefore the potential at the plate equals zero. This observation allows to alternate the current setup, so there are exclusively homogeneous boundary conditions and the Kirchhoff's integral can be applied. The idea is, to mirror the electret at the plane of the plate and assume the mirrored charge ' $q$ to be the opposite charge to $q$ as shown in Fig. 5. The influence of the physical electret charge $q$ and the introduced artificial opposite charge ' $q$ cancel each other resulting in a constant zero potential at the plane of the plate. Doing so, the boundary condition of a constant field potential at the plane of the plate is implicitly satisfied and the plate as an inhomogeneous boundary condition can be left out. Thus, we obtain an alternated setup with homogeneous boundary conditions and the Kirchhoff's integral can be applied (Küpfmüller et al., 2013, p. 157). 


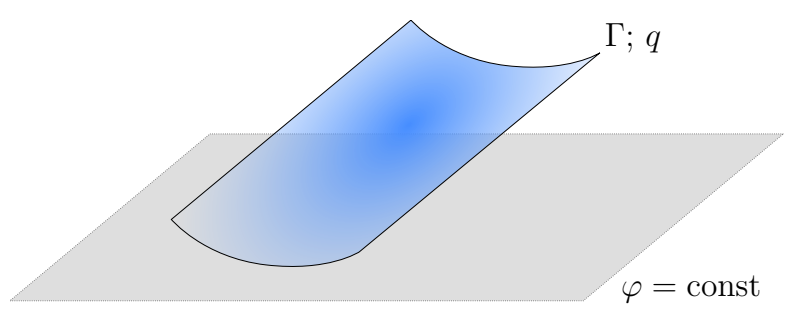

Figure 4. Inhomogeneous electrostatic field problem to solve, with a constant potential boundary condition at the plate.

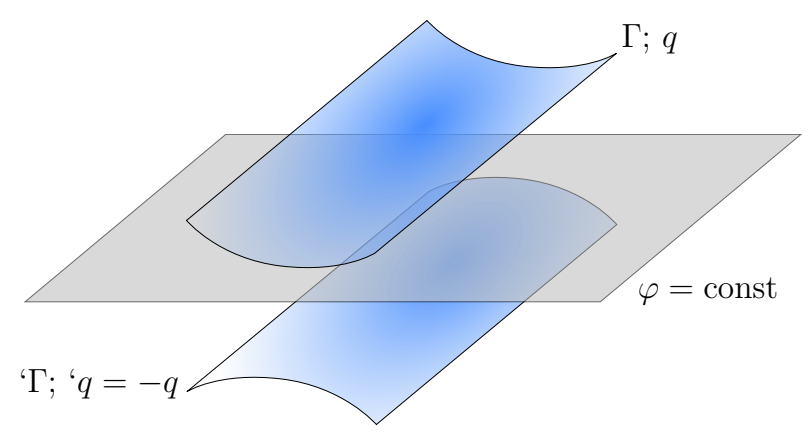

Figure 5. Homogeneous electrostatic field problem, with a constant potential at the plane described by the plate.

Furthermore, the electret consists of a thin foil, so it is possible to simplify the integration in Eq. (18) to

$\varphi(\boldsymbol{r})=\frac{1}{4 \pi \varepsilon} \iint_{\widetilde{A}} \frac{\varrho(\widetilde{\boldsymbol{r}})}{\|\boldsymbol{r}-\widetilde{\boldsymbol{r}}\|} \mathrm{d} \widetilde{A}$.

\section{Approximation of the analytic solution}

Although, an analytic solution for the sensor model has been derived, there still exists the problem of integrating the charge over a complex geometry. For the modeling purpose it is easier to use a discretization of the electret and translate the integration into a sum.

For the discretization, the electret is divided into regular small part-areas $\Delta \widetilde{A}$ with a part-charge $\Delta \widetilde{q}$ as shown in Fig. 6. The integration in Eq. (19) is translated into a sum

$\varphi(\mathbf{r})=\frac{\Delta \widetilde{q} \Delta \widetilde{A}}{4 \pi \varepsilon} \sum_{\Delta \widetilde{A}} \frac{1}{\|\boldsymbol{r}-\widetilde{\boldsymbol{r}}\|}$

and is applied for

$\left.\varphi(x, z)\right|_{y=\text { const }} \quad \forall y \in[-h ;+\infty)$.

This restriction to the solution is based on the fact, that in reality the space below the ideal conducting plate is shielded from the influence of the electric charge by the plate itself.

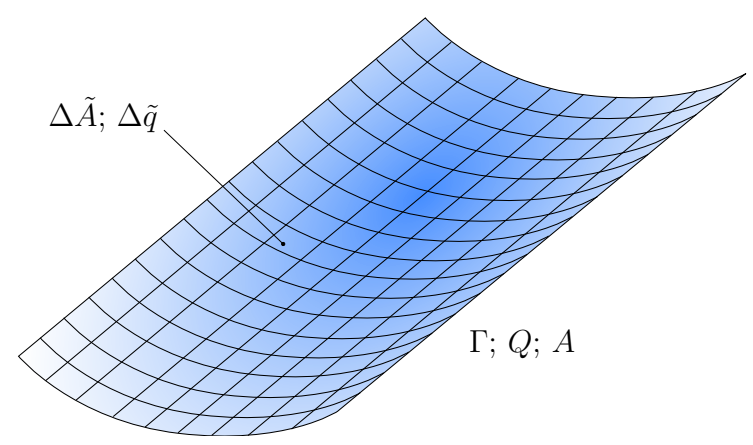

Figure 6. Regular discretization of the electret.

\section{Induced charge on the plane}

As described in Sect. 2 the induced charge $Q$ on the plane depends on the torque load $M_{\mathrm{t}}$. This induced charge can be calculated using the results in Sect. 6 for the potential $\varphi$ by applying the divergence theorem

$Q=\int_{\mathcal{V}} \operatorname{div} \boldsymbol{D} \mathrm{d} V=\oint_{\mathcal{A}} \boldsymbol{D} \cdot \boldsymbol{n} \mathrm{d} A$

and using the constitutive equation

$\boldsymbol{D}=-\varepsilon \operatorname{grad} \varphi$.

Assuming only a static electric field, the field in the immediate proximity of the plate is perpendicular to the ideal conducting plate and the gradient at the plate is defined by

$\boldsymbol{D}=-\varepsilon \lim _{\Delta h \rightarrow 0} \frac{\left.\varphi(x, z)\right|_{y=-h}-\left.\varphi(x, z)\right|_{y=-h+\Delta h}}{\Delta h} \boldsymbol{e}_{y}$.

Similar to the discretization of the electret, it is possible to sum over the small part-areas of the ideal conducting plane as follows

$Q=\sum_{\Delta \widetilde{A}} \boldsymbol{D} \boldsymbol{e}_{y}^{\top} \Delta \widetilde{A}$.

Although, the ideal conducting plate is assumed to be infinite, it is accurate enough, to sum over a finite area. The areas further away from the electric charge do not contribute to the overall induced charge on the plate, because the potential field decreases over the distance.

\section{Computation results}

In Fig. 7 the potential is shown in two different heights over the electret. It becomes apparent that the potential reaches its major values, where it is closer to the electret.

Figure 8 represents the charge density on the ideal conducting plate. 
(a)

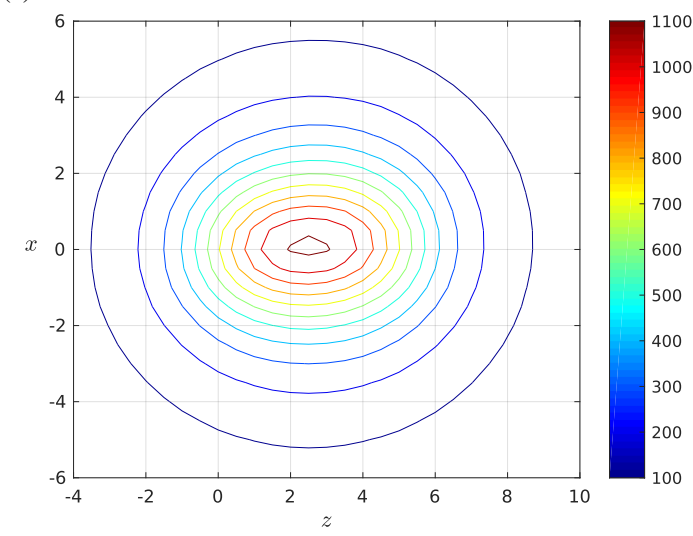

(b)

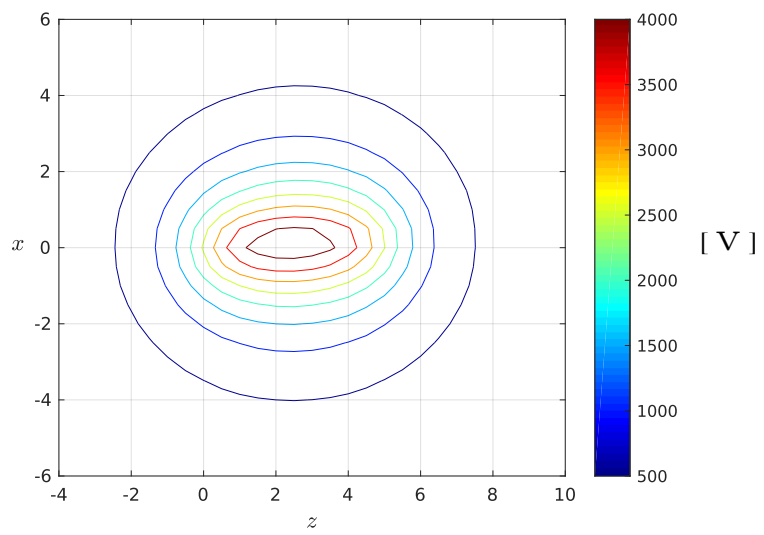

Figure 7. Potential planes $\left.\varphi(x, z)\right|_{y=\text { const }}$ in two different heights. (a) $y=-h+\frac{1}{4} h$. (b) $y=-h+\frac{1}{2} h$.

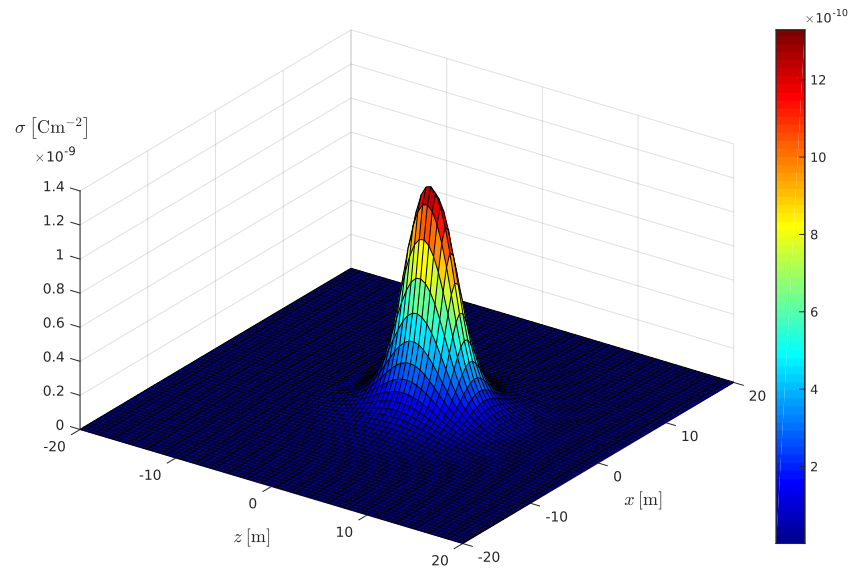

Figure 8. Charge density $\sigma(x, z)$ as the charge per area.

The quantity of the induced charge is calculated as described in Sect. 8. This charge can be measured and depends on the torque load, which is applied to the beam. Therefore, a characteristic curve of the sensor is derived. For this purpose, the charge for different torque loads is simulated and recored. Finally, the characteristic curve is approximated by a polynomial function of 2 nd order. The results are shown in Fig. 9.

\section{Conclusions}

As one can see in Fig. 9, the maximum induced charge occurs, when there is no torque load being applied to the beam. At that configuration the electret is closest to the plate. By increasing the load, the electret is being moved further away from the ideal conducting plate and one can observe a decrease in the induced charge on the plate. The numerical analysis of the measuring setup also shows, that it is only possible to conclude the absolute value of the torque load from the

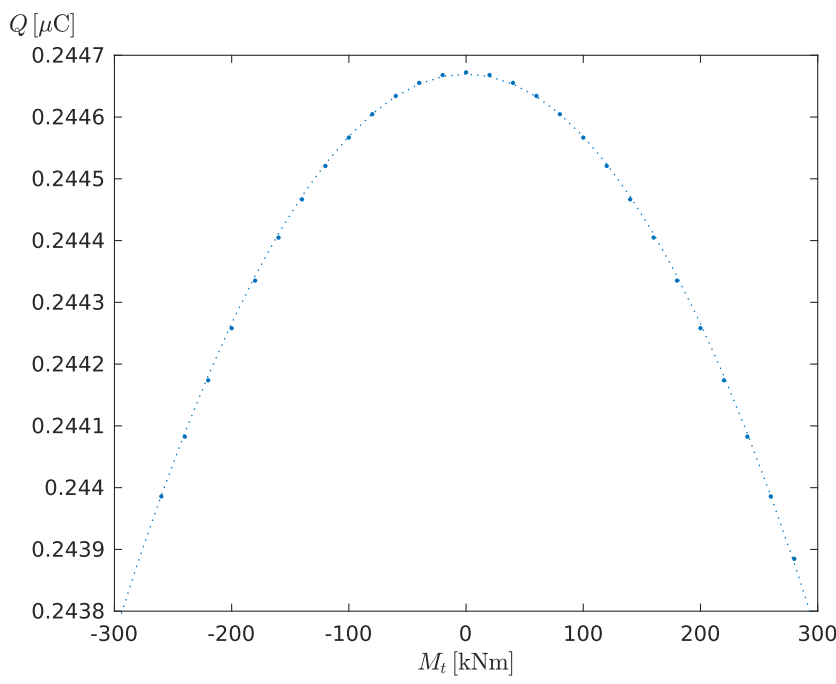

Figure 9. The characteristic curve of the sensor fitted by a polynomial function of 2nd order $Q_{\mathrm{p}}\left(M_{\mathrm{t}}\right)$.

charge induced on the ideal conducting plate. This result is in line with the symmetry of the system.

Furthermore, an expected sensitivity deficiency is being observed. This is bound to the small quasi-permanent charge an electret holds and the distance to the ideal conducting plate.

Interestingly, the polynomial function of 2 nd order $Q_{\mathrm{p}}\left(M_{\mathrm{t}}\right)$ fitted by the method of least squares fits the characteristic curve well. Introducing an objective error metric as a median error

$e_{Q}=\frac{\sum_{i=1}^{n}\left\|Q_{i}\left(M_{i}\right)-Q_{\mathrm{p}}\left(M_{i}\right)\right\|_{2}}{n}$

where $Q_{i}\left(M_{i}\right)$ is the measured charge for an applied torque $M_{i}$ and $Q_{\mathrm{p}}\left(M_{i}\right)$ is the value of the polynomial function at that point; assuming there are $n$ different probed torque 
loads, for the characteristic in Fig. 9 the error adds up to $5.31 \times 10^{-12}$. As the dimension of the charge is of order $2.44 \times 10^{-7}$, it is reasonable to deduce a quadratic trend of the sensor, when linear assumptions are met.

\section{Summary}

In this contribution an analysis of an electrostatic sensor for torque load measurements is presented. Due to the necessity of describing the position of the charge in the physical space, a geometrical and mechanical model based on the St. Vénant torsion theory is described. In order to solve the well known Poisson equation for this problem, the Kirchhoff's integral theorem and an alternated model are used. Further, a discretization is used to solve the integral and also to calculate the induced charge numerically. As shown, the proposed sensor has only a low sensitivity. Considering the dimensions and the sensitivity issues of the measuring setup, the practical application is not optimal. Nevertheless, in the further research an improvement of the sensitivity will be considered.

Data availability. No data sets were used in this article.

Competing interests. The authors declare that they have no conflict of interest.
The publication of this article was funded by the open-access fund of Leibniz Universität Hannover.

Edited by: J. Anders

Reviewed by: two anonymous referees

\section{References}

Kapralov, V. M. and Fesenko, N. I.: Accuracy in measuring mechanical stresses (strains) with resistance strain gauges, Strength Mater., 16, 1209, https://doi.org/10.1007/BF01530302, 1984.

Küpfmüller, K., Mathis, W., and Reibiger, A.: Theoretische Elektrotechnik: Eine Einführung, Springerverlag Berlin Heidelberg, 2013.

Szabó, I.: Höhere Technische Mechanik: nach Vorlesungen, Springerverlag Berlin Heidelberg, 2001.

Turner, J.: The development of a thick-film non-contact shaft torque sensor for automotive applications, Department of Mechanical Engineering, University of Southampton, Highfield, Southampton, UK, 1988.

Wolffenbuttel, R. F. and Foerster, J. A.: Noncontact Capacitive Torque Sensor For Use on a Rotating Axle, IEEE T. Instrum. Meas., 39, 1008-1013, https://doi.org/10.1109/19.65816, 1990. 